\title{
Relapsing Polychondritis: An Otolaryngologist's Perspective
}

\author{
${ }^{1}$ Kamran A Syed, ${ }^{2} \mathrm{P}$ Naina, ${ }^{3}$ Sheeja S John, ${ }^{4}$ Ajoy M Varghese
}

\begin{abstract}
Relapsing polychondritis (RP) is an uncommon autoimmune disease with varying clinical presentations. Relapsing polychondritis frequently involves the ear, nose, and throat cartilages, often causing subtle and episodic symptoms that can pose a diagnostic challenge to the otolaryngologist. A high index of suspicion is needed to identify these subtle signs leading to early diagnosis. Here, we report a case of RP presenting with hoarseness of voice; we reviewed the literature and clinical signs discussed from an otolaryngologist's perspective. Greater awareness about its episodic and myriad presentation is needed for early diagnosis and management of this uncommon clinical condition.
\end{abstract}

Keywords: Relapsing polychondritis, Saddle nose, Tracheal stenosis.

How to cite this article: Syed KA, Naina P, John SS, Varghese AM. Relapsing Polychondritis: An Otolaryngologist's Perspective. Int J Otorhinolaryngol Clin 2017;9(1):17-20.

Source of support: Nil

Conflict of interest: None

\section{INTRODUCTION}

Relapsing polychondritis (RP) is a rare, progressive, chronic, multisystem disorder characterized by recurrent inflammation of the cartilages of the ear, nose, larynx, tracheobronchial tree, and the cartilages of large joints. ${ }^{1}$ Dermatological, cardiac, renal, neurological, and ocular involvement may also be present.

The variable, episodic expression of clinical features over time often makes the diagnosis difficult to establish. This may present with episodic dyspnea, cough, wheeze, stridor, and hoarseness of voice. ${ }^{2}$ There is a varied incidence of airway involvement reported in the literature. Airway involvement is seen in nearly half the cases of RP in Caucasians, ${ }^{3,4}$ whereas studies from India have reported prevalence ranging from 12 to $43 \%{ }^{5,6}$

\footnotetext{
${ }^{1,2}$ Assistant Professor, ${ }^{3,4}$ Professor

1,2,4 Department of ENT - Unit 2, Christian Medical College Vellore, Tamil Nadu, India

${ }^{3}$ Department of Ophthalmology, Christian Medical College Vellore, Tamil Nadu, India

Corresponding Author: Kamran A Syed, Assistant Professor Department of ENT - Unit 2, Christian Medical College, Vellore Tamil Nadu, India, Phone: +9104162282798, e-mail: skamrann @gmail.com
}

Airway involvement is the most serious manifestation of the disease and can be prevented with early detection. ${ }^{6}$ Therefore, early diagnosis and timely intervention are imperative. We report a case of RP with hoarseness of voice, which had been misdiagnosed as "bronchial asthma" for 3 years.

\section{CASE REPORT}

A 36-year-old man was referred to the Voice Clinic at a tertiary institution in South India for evaluation of progressive hoarseness. He was on treatment for wheezing and dyspnea, diagnosed as bronchial asthma for the past 3 years with no significant relief of symptoms. Further questioning elicited history of recurrent episodes of pain and swelling of both pinnae and polyarthralgia for the past 9 years, for which he had been given symptomatic treatment by local doctors. On examination, he had a saddle nose deformity (Fig. 1), with subacute chondritis in both ears. Laryngeal examination revealed thickening of both true and false vocal folds with minimal airway compromise.

Contrast-enhanced axial computed tomography (CT) scan of the neck and thorax showed marked thickening of the aryepiglottic folds and the true vocal cords, causing glottic stenosis. There was marked thickening of the cricoid cartilage with characteristic "train-track" calcification and subglottic narrowing (Fig. 2). The arytenoids and thyroid cartilages were also ossified to a degree not expected for his age (Fig. 3). There was tracheobronchial wall thickening with luminal narrowing

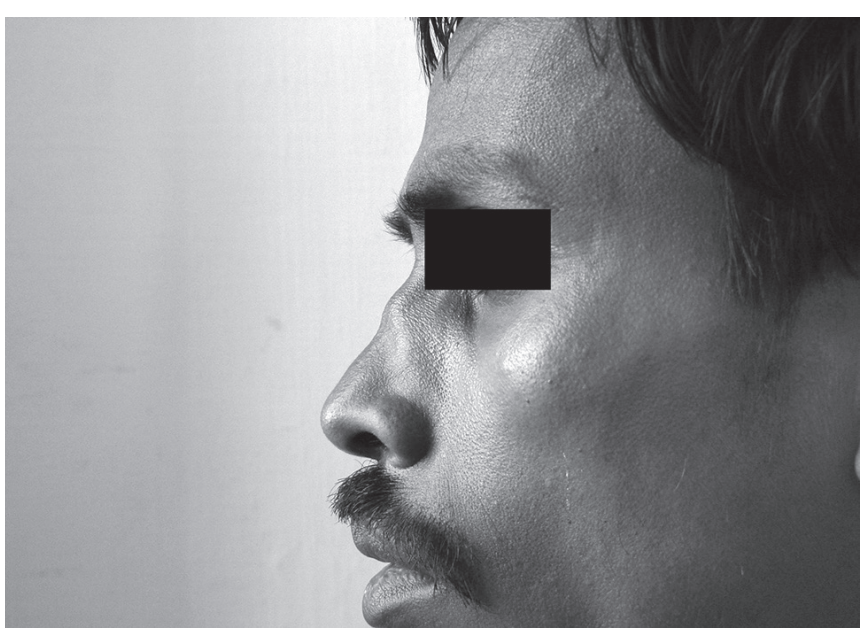

Fig. 1: Saddle nose deformity 


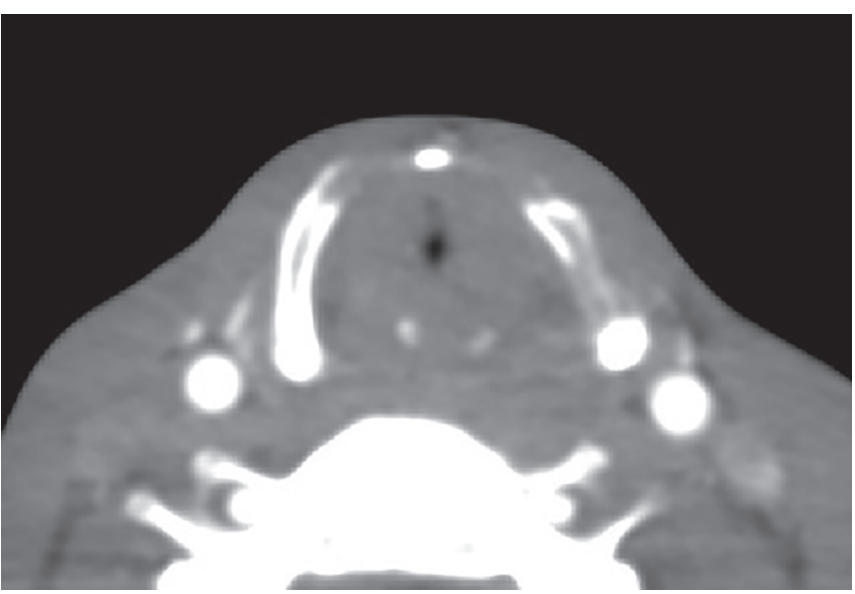

Fig. 2: Contrast-enhanced axial CT neck showing characteristic "train-track" calcification of cricoid cartilage

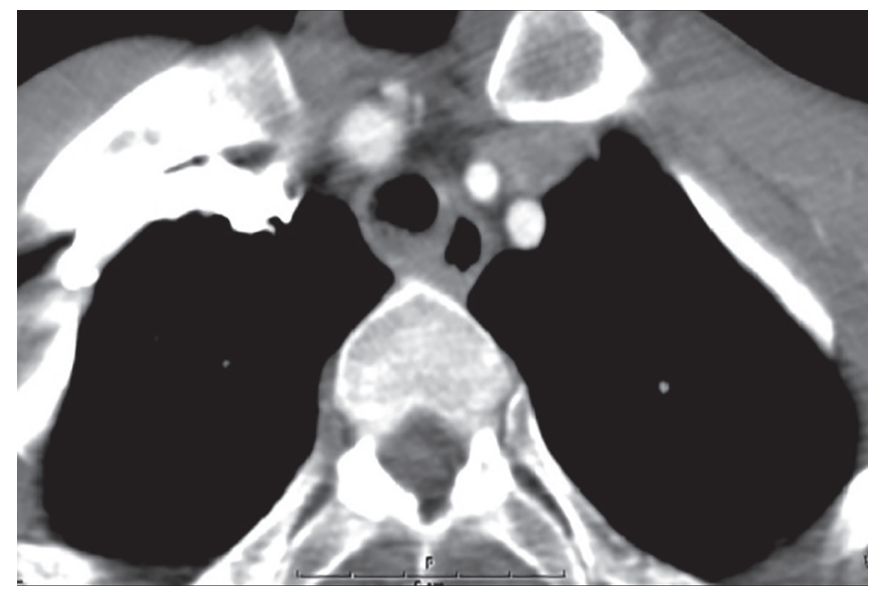

Fig. 4: Contrast-enhanced axial CT neck showing early tracheal calcification and thickening

and early calcification of tracheal cartilage with sparing of the posterior membrane (Fig. 4).

Routine laboratory test results were within normal limits. Antinuclear antibody, rheumatoid factor, C-reactive protein, and antineutrophil cytoplasmic antibodies were negative; Venereal Disease Research Laboratory test was nonreactive; and serum angiotensin-converting enzyme levels were within normal limits. X-rays of the small and large joints of the upper and lower limbs were normal. As the patient satisfied the criteria for diagnosis of RP (McAdam's diagnostic criteria, as modified by Damiani and Levine) (Table 1), ${ }^{3,7}$ he was started on high-dose oral prednisolone and cyclophosphamide. He responded well to treatment and is on follow-up.

\section{DISCUSSION}

Relapsing polychondritis is a rare disease, with varying clinical presentations, thus posing a diagnostic challenge. Although the pathogenesis of the disease is not yet fully understood, recent evidence is suggestive of

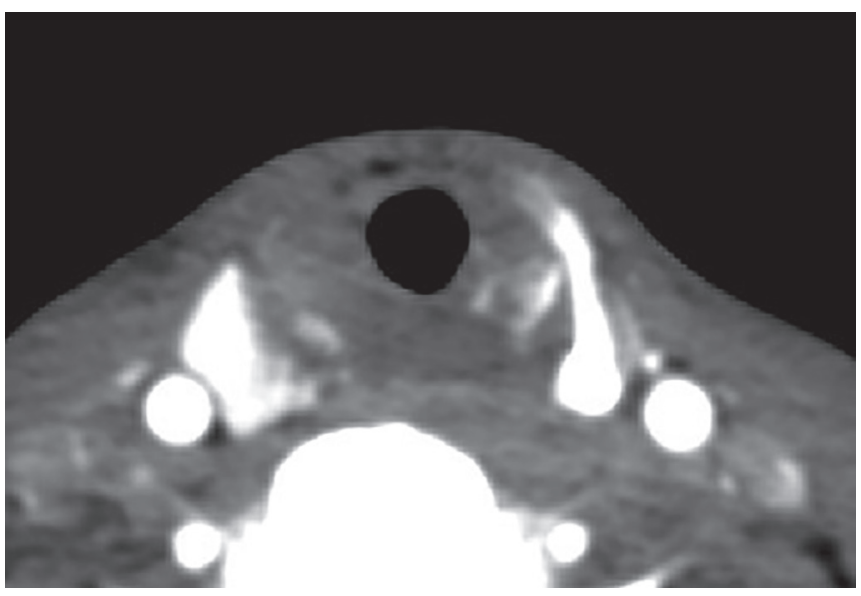

Fig. 3: Contrast-enhanced axial CT neck showing thyroid and arytenoid cartilage calcification with glottic narrowing to a degree not expected in this age group

a Th-1-mediated autoimmune response against type 2 collagen and matrilin. ${ }^{8}$ As no specific tests are available, the disease is usually diagnosed on clinical grounds. ${ }^{1}$ The definitive diagnosis of RP is based on McAdam's diagnostic criteria, as modified by Damiani and Levine (Table 1). ${ }^{3,7}$

Due to the episodic nature of the inflammatory manifestations and the multisystem involvement, the disease is often misdiagnosed. Relapsing polychondritis can present to various specialists, from primary care physicians to otolaryngologists, ophthalmologists, and rheumatologists. Collaboration and interdisciplinary cooperation between various specialties is the way forward in modern medicine in

Table 1: Diagnostic criteria for RP

\begin{tabular}{|c|c|c|}
\hline $\begin{array}{l}\text { Author (date) } \\
\text { and criteria }\end{array}$ & & nical features \\
\hline \multirow{6}{*}{$\begin{array}{l}\text { McAdam et al } \\
\text { The presence of } \\
\text { three or more of } \\
\text { the given clinical } \\
\text { features }\end{array}$} & 1 & Recurrent chondritis of both auricles \\
\hline & 2 & Nonerosive inflammatory arthritis \\
\hline & 3 & Chondritis of the nasal cartilages \\
\hline & 4 & $\begin{array}{l}\text { Ocular inflammation including } \\
\text { conjunctivitis, keratitis, scleritis, } \\
\text { episcleritis, and/ or uveitis }\end{array}$ \\
\hline & 5 & $\begin{array}{l}\text { Chondritis of the respiratory tract } \\
\text { involving the laryngeal and/or tracheal } \\
\text { cartilages }\end{array}$ \\
\hline & 6 & $\begin{array}{l}\text { Cochlear and/or vestibular damage } \\
\text { manifested by sensorineural hearing loss, } \\
\text { tinnitus, and/or vertigo }\end{array}$ \\
\hline \multirow{3}{*}{$\begin{array}{l}\text { Damiani and } \\
\text { Levine (1979) } \\
\text { The presence } \\
\text { of any one } \\
\text { of the given } \\
\text { combinations }\end{array}$} & 1 & $\begin{array}{l}\text { At least three or more diagnostic criteria } \\
\text { (McAdam's), histological confirmation not } \\
\text { necessary }\end{array}$ \\
\hline & 2 & $\begin{array}{l}\text { One or more of McAdam's signs with a } \\
\text { positive histological confirmation }\end{array}$ \\
\hline & 3 & $\begin{array}{l}\text { Chondritis in two or more separate } \\
\text { anatomical locations with response to } \\
\text { steroids and/or dapsone }\end{array}$ \\
\hline
\end{tabular}


understanding and managing both common and uncommon conditions. ${ }^{9}$

The mean delay from the time medical attention was sought until a definitive diagnosis was made has varied from 2.9 years in earlier case series to 20 months in recent reports. ${ }^{4,6}$ This delay reflects the diagnostic uncertainty that is characteristic of the disease. The delay was longer than 1 year in $68 \%$ of the patients, and one-third of them needed to see five or more physicians before a definitive diagnosis of RP was made. ${ }^{4}$ In our case, although many of the classical clinical features of RP were there on presentation, the diagnosis had been totally missed, and the patient had been on treatment for "bronchial asthma" for 3 years.

Pinna involvement with auricular chondritis is the commonest presentation reported in literature. ${ }^{10}$ Inner ear involvement manifesting as sensorineural hearing loss was absent. Saddling of nose with nasal chondritis is the commonest nasal finding, which may be present initially or may manifest at a later stage ${ }^{11}$ In our patient, saddling of nose along with subacute aural chondritis was seen at presentation. Involvement of the cartilaginous structures of the respiratory tract is one of the most serious complications of RP and accounts for up to $50 \%$ of the deaths associated with the disease. ${ }^{12}$ The mechanisms of airway obstruction include: (1) Inflammatory swelling in the active stage causing narrowing of airway; (2) progressive destruction of the respiratory cartilages causing dynamic collapse of the airway in the earlier stages, and (3) cicatricial contraction by fibrous tissue at a later stage. ${ }^{2}$

Tracheobronchial luminal narrowing is not specific to $\mathrm{RP}^{13}$ The differential diagnoses include tracheopathia osteochondroplastica, granulomatosis with polyangiitis, sarcoidosis, and diffuse amyloidosis, which were ruled out in our patient. Computerized tomography scan is the most useful investigation to evaluate the cartilaginous and soft tissue involvement in the tracheobronchial airway. ${ }^{14}$

The disease usually has a fluctuating, but progressive course, and the mainstay of treatment is suppression of autoimmunity and inflammation. ${ }^{5}$ However, the outcome may be difficult to predict. The severity and chronicity of the disease, as well as the extent of multisystemic involvement, determine the choice of pharmacological agent used, as well as the variable outcomes that are reported in literature. ${ }^{1,2,4,5}$ Often, the choice of treatment is individualized. Nonsteroidal anti-inflammatory drugs form the first line of management in uncomplicated cases, with only nasal or auricular cartilage involvement. Steroids are often used in patients with more serious and life-threatening symptoms, or with multisystemic involvement. ${ }^{5}$ Steroid-sparing agents that have been found effective in RP include azathioprine, cyclophosphamide, methotrexate, dapsone, and cyclosporine, but outcomes are difficult to compare. ${ }^{2}$ High-dose oral prednisolone therapy is indicated in respiratory tract involvement in RP. Intravenous pulse methyl prednisolone may be needed in acute airway obstruction. ${ }^{2}$ Tracheostomy is indicated in severe, localized subglottic involvement, while stenting of the respiratory tract can alleviate more widespread tracheobronchial disease. ${ }^{12}$ However, our patient responded very well to high-dose steroids and cyclophosphamide and, hence, did not require tracheostomy or stenting.

In conclusion, RP is a rare disease, which often presents in an enigmatic fashion, and is frequently misdiagnosed. Recurrent inflammation of aural cartilage, saddling of nose, hoarseness of voice, or dyspnea along with episodic joint pain should raise strong clinical suspicion and warrants further evaluation. Airway involvement in the disease is serious and can be potentially fatal. A patient with RP may present to an otolaryngologist primarily or referred from different specialists. Greater awareness and early recognition of the signs of this disease are imperative for early diagnosis and appropriate management of the disease.

\section{REFERENCES}

1. Sharma A, Gnanapandithan K, Sharma K, Sharma S. Relapsing polychondritis: a review. Clin Rheumatol 2013 Nov;32(11):1575-1583.

2. Rafeq S, Trentham D, Ernst A. Pulmonary manifestations of relapsing polychondritis. Clin Chest Med 2010 Sep;31(3):513-518.

3. McAdam LP, O'Hanlan MA, Bluestone R, Pearson CM. Relapsing polychondritis: prospective study of 23 patients and a review of the literature. Medicine (Baltimore) 1976 May;55(3):193-215.

4. Trentham DE, Le CH. Relapsing polychondritis. Ann Intern Med 1998 Jul;129(2):114-122.

5. Sharma A, Law AD, Bambery P, Sagar V, Wanchu A, Dhir V, Vijayvergiya R, Sharma K, Gupta A, Panda NK, et al. Relapsing polychondritis: clinical presentations, disease activity and outcomes. Orphanet J Rare Dis 2014 Dec;9:198.

6. Ananthakrishna R, Goel R, Padhan P, Mathew J, Danda D. Relapsing polychondritis—case series from South India. Clin Rheumatol 2009 Jun;28(Suppl 1):7-10.

7. Damiani JM, Levine HL. Relapsing polychondritis - report of ten cases. Laryngoscope 1979 Jun;89:929-946.

8. Arnaud L, Mathian A, Haroche J, Gorochov G, Amoura Z. Pathogenesis of relapsing polychondritis: a 2013 update. Autoimmun Rev 2014 Feb;13(2):90-95.

9. Danda D. Collaboration, the other name for research. Int J Rheum Dis 2014 Jan;17(1):4.

10. Sharma A, Bambery P, Wanchu A, Sharma YP, Panda NK, Gupta A, Singh S. Relapsing polychondritis in North India: 
a report of 10 patients. Scand J Rheumatol 2007 Nov-Dec; 36(6):462-465.

11. Isaak BL, Liesegang TJ, Michet CJ. Ocular and systemic findings in relapsing polychondritis. Ophthalmology 1986 May;93(5):681-689.

12. Buscatti IM, Giacomin MFA, Silva MFC, Campos LMA, Sallum AME, Silva CA. Laryngotracheal stenosis requiring emergency tracheostomy as the first manifestation of childhood-relapsing polychondritis. Acta Rheumatol Port 2013 Jul-Sep;38(3):208-211.

13. Grenier PA, Beigelman-Aubry C, Brillet P-Y. Nonneoplastic tracheal and bronchial stenoses. Radiol Clin North Am 2009 Mar;47(2):243-260

14. Anchan SV, Garag SS, Arunkumar JS, Prasad KC, Sharma PV. An interesting case of relapsing polychondritis in a young girl. J Clin Diagn Res 2013 Dec;7(12):2976-2977. 